\title{
Etude des phénomènes de réfraction et de diffraction avec un modèle d'approche de la houle en coordonnées curvilignes
}

\author{
Nicolas JARRY ${ }^{1}$, Jean BOUGIS ${ }^{2}$, David LAJOIE ${ }^{3}$, Vincent REY ${ }^{4}$ \\ ${ }^{1}$ Doctorant, ${ }^{3}$ Ingénieur, ACRI-IN, 260 Route du Pin Montard, BP 234, Sophia- \\ Antipolis, France \\ nicolas.jarry@acri-in.fr, david.lajoie@acri-in.fr \\ ${ }^{2}$ Ingénieur conseil, 34 Chemin du Moulin, 06650 Opio, France \\ jean.bougis@wanadoo.fr \\ ${ }^{4}$ Professeur, Université de Toulon et du Var, UMR 6017 - LSEET-LEPI, \\ 83957 La Garde Cedex, France \\ rey@univ-tln.fr
}

\section{Résumé :}

Le modèle présenté ici suit la propagation de la houle dans les coordonnées curvilignes formées par les rayons et les crêtes (BOUGIS, 2004) évitant ainsi les limitations angulaires liées à la parabolisation de l'équation de Berkhoff sur un axe privilégié. La méthode consiste à construire un maillage en réfraction pure d'abord, puis à le modifier en tenant compte de la diffraction par un processus itératif. Les influences de la réfraction et de la diffraction, calculées de façon combinée, peuvent néanmoins être analysées de manière découplée. Le modèle est ensuite confronté à REFDIF en houle régulière et à SWAN en houle irrégulière et montre des résultats intéressants.

\begin{abstract}
:
The model presented here follows wave propagation in curvilinear coordinates given by rays and crests, (BOUGIS 2004), avoiding angle limitations linked to the parabolic approximation of mild-slope equation on a privileged axis. The method consists of building the meshing in refraction first, and then of modifying it in taking into account diffraction effects by an iterative process. Refraction and diffraction influences, calculated in a combined way, can nevertheless be analysed separately. The model is then compared to REFDIF in monochromatic waves, and to SWAN in irregular waves, and shows interesting results.
\end{abstract}




\section{Mots-clés :}

Houle - Réfraction - Diffraction - Curviligne - Rayons - Crêtes

\section{$1 \quad$ Introduction}

Les modèles d'approche de la houle sont destinés à suivre les modifications que subit la houle au cours de sa propagation. On distingue les modèles à phase moyennée et les modèles à résolution de phase. HOLTHUIJSEN et al. (2003) ont introduit de la diffraction dans un modèle spectral par une approximation de réfraction-diffraction à phase découplée. Elle est basée sur l'équation en pente douce et exprimée en terme de changement directionnel des composantes de chaque vague dans leur spectre bidimensionnel, en omettant toute information sur la phase. C'est sur le même principe qu'un paramètre de diffraction a été ajouté dans un modèle à résolution de phase, dans le but de résoudre l'équation en pente douce sur un maillage construit à l'avancement en réfraction-diffraction.

En effet, BOUGIS (2004) a développé un modèle d'approche de la houle par une méthode de réfraction-diffraction en coordonnées curvilignes formées par les rayons et les crêtes. Lorsque la houle tourne, la dérivée seconde de la hauteur est ainsi toujours calculée le long des crêtes, et les limitations angulaires liées à la parabolisation de l'équation de Berkhoff sur un axe privilégié (RADDER, 1979), sont alors éliminées. Le processus est itératif puisque la métrique n'est pas connue a priori et les solutions propagatives sont recherchées en conservant la dérivée seconde de la hauteur dans le sens de la propagation. La méthode présentée ici, basée sur le modèle de BOUGIS, consiste à construire un maillage en réfraction pure d'abord, puis à le modifier grâce à un paramètre de diffraction, par un processus itératif. Ce paramètre modifie alors les caractéristiques des vagues et donc la métrique jusqu'à la convergence du modèle.

\section{Equation de Berkhoff en coordonnées curvilignes orthogonales}

\subsection{Equation de réfraction diffraction}

En présence d'un courant indépendant du temps et uniforme sur la profondeur, induisant une dénivelée de la surface libre $\zeta$, l'équation de réfraction-diffraction combinée introduite par BERKHOFF (1972) s'écrit :

$$
\operatorname{div}\left(C C_{g} \overrightarrow{g r a d} H\right)-g \cdot \overrightarrow{g r a d} \zeta \cdot \overrightarrow{g r a d} H+\left(1+i v_{t}\right) k^{2} C C_{g} H=0
$$

Où le terme $\left(i n_{t}\right)$ traduit les pertes d'énergie par dissipation, $H$ la représentation complexe de la hauteur crête à creux de l'élévation de la surface libre à la pulsation $\omega, C$ la vitesse de phase, $C_{g}$ la vitesse de groupe et $k$ le nombre d'onde. 


\subsection{Equation en coordonnées curvilignes}

On considère le système de coordonnées curvilignes orthogonales dont les axes $x$ et $\eta$ coïncident respectivement avec le rayon local et la crête locale de la houle ; $e_{1}$ et $e_{2}$ les facteurs d'échelle associés aux systèmes de coordonnées :

$$
e_{1}^{2}=\left(\frac{\partial x}{\partial \xi}\right)^{2}+\left(\frac{\partial y}{\partial \xi}\right)^{2} \text { et } e_{2}^{2}=\left(\frac{\partial x}{\partial \eta}\right)^{2}+\left(\frac{\partial y}{\partial \eta}\right)^{2}
$$

En exploitant les expressions des opérateurs vectoriels et après développement et séparation des termes, on obtient l'équation de réfraction-diffraction suivante :

$$
\begin{aligned}
& \frac{C C g}{e_{1}^{2}} \frac{\partial^{2} H}{\partial \xi^{2}}+\frac{C C g}{e_{2}^{2}} \frac{\partial^{2} H}{\partial \eta^{2}}+\left[\frac{C C_{g}}{e_{1} e_{2}} \frac{\partial}{\partial \xi} \frac{e_{2}}{e_{1}}+\frac{1}{e_{1}^{2}}\left(\frac{\partial C C_{g}}{\partial \xi}-g \frac{\partial \zeta}{\partial \xi}\right)\right] \frac{\partial H}{\partial \xi}+ \\
& {\left[\frac{C C_{g}}{e_{1} e_{2}} \frac{\partial}{\partial \eta} \frac{e_{1}}{e_{2}}+\frac{1}{e_{2}^{2}}\left(\frac{\partial C C_{g}}{\partial \eta}-g \frac{\partial \zeta}{\partial \eta}\right)\right] \frac{\partial H}{\partial \eta}+k^{2} C C g\left(1+i v_{t}\right) H=0}
\end{aligned}
$$

\subsection{Equation de l'enveloppe de la houle}

La hauteur crête à creux $H$ peut s'écrire en dissociant l'enveloppe des hauteurs $\hat{H}$ et sa phase $S$ tel que $H=\hat{H} e^{i s}$. Par construction, on admet dans un premier temps la relation suivante valable uniquement en réfraction pure :

$\vec{k}=\overrightarrow{\operatorname{grad}} S$

L'équation de Berkhoff généralisée en coordonnées curvilignes orthogonales en fonction de l'enveloppe de la houle devient donc :

$$
\begin{aligned}
& \frac{C C g}{e_{1}^{2}} \frac{\partial^{2} \hat{H}}{\partial \xi^{2}}+\frac{C C g}{e_{2}^{2}} \frac{\partial^{2} \hat{H}}{\partial \eta^{2}} \\
& +\left[2 i k e_{1} \frac{C C_{g}}{e_{1}^{2}}+\frac{C C_{g}}{e_{1} e_{2}} \frac{\partial}{\partial \xi}\left(\frac{e_{2}}{e_{1}}\right)+\frac{1}{e_{1}^{2}}\left(\frac{\partial C C_{g}}{\partial \xi}-g \frac{\partial \zeta}{\partial \xi}\right)\right] \frac{\partial \hat{H}}{\partial \xi} \\
& +\left[\frac{C C_{g}}{e_{1} e_{2}} \frac{\partial}{\partial \eta}\left(\frac{e_{1}}{e_{2}}\right)+\frac{1}{e_{2}^{2}}\left(\frac{\partial C C_{g}}{\partial \eta}-g \frac{\partial \zeta}{\partial \eta}\right)\right] \frac{\partial \hat{H}}{\partial \eta} \\
& +\left[i v_{t} k^{2} C C_{g}+\frac{i C C_{g}}{e_{1}} \frac{\partial k}{\partial \xi}+i k\left(\frac{C C_{g}}{e_{1} e_{2}} \frac{\partial e_{2}}{\partial \xi}+\frac{1}{e_{1}}\left(\frac{\partial C C_{g}}{\partial \xi}-g \frac{\partial \zeta}{\partial \xi}\right)\right)\right] \hat{H}=0
\end{aligned}
$$




\section{$3 \quad$ Insertion d'un paramètre de diffraction}

L'équation de réfraction diffraction (1) en absence de courant (de manière à soulager l'écriture) et en fonction de l'enveloppe des hauteurs $\hat{H}$ et de la phase $S$ a pour expression :

$$
\operatorname{div}\left(C C_{g} \overrightarrow{g r a d} \hat{H} e^{i s}\right)+k^{2} C C_{g} \hat{H} e^{i s}=0
$$

En séparant partie réelle et partie imaginaire et après manipulation des équations, on parvient à:

$$
\begin{aligned}
& (\overrightarrow{\operatorname{grad} S})^{2}=k^{2}+\frac{\operatorname{div}\left(C C_{g} \overrightarrow{\operatorname{grad}} \hat{H}\right)}{C C_{g} \hat{H}} \\
& \operatorname{div}\left(C C_{g} \hat{H}^{2} \overrightarrow{\operatorname{grad} S}\right)=0
\end{aligned}
$$

En réfraction pure où la variation de l'amplitude est considérée comme négligeable, l'équation (7) conduit à l'équation eïkonale (4). Mais lorsque le phénomène de diffraction devient important, le terme $\operatorname{div}\left(C C_{g} \overrightarrow{g r a d} \hat{H}\right) / C C_{g} \hat{H}$ ne peut être négligé si bien que $k$ n'est plus directement assimilable au nombre d'onde d'une onde progressive. En posant :

$$
\overrightarrow{k^{\prime}}=\overrightarrow{\operatorname{grad}} S
$$

Les deux équations (7) et (8) s'écrivent alors (HOLTUIJSEN et al., 2003) :

$$
\begin{aligned}
& k^{\prime 2}=k^{2}+\frac{\operatorname{div}\left(C C_{g} \overrightarrow{g r a d} \hat{H}\right)}{C C_{g} \hat{H}} \\
& \operatorname{div}\left(C C_{g} \hat{H}^{2} \overrightarrow{k^{\prime}}\right)=\operatorname{div}\left(\frac{\omega}{k} \hat{H}^{2} C_{g} \overrightarrow{k^{\prime}}\right)=0 \text { d'où } \operatorname{div}\left(\hat{H}^{2} C_{g} \frac{\overrightarrow{k^{\prime}}}{k}\right)=0
\end{aligned}
$$

En présence de diffraction, une vague se propage à la vitesse de phase corrigée $C^{\prime}$ telle que $k^{\prime}=\omega / C^{\prime}$. On pose ainsi le paramètre de diffraction $\delta_{H}$ tel que :

$$
\delta_{H}=\frac{\operatorname{div}\left(C C_{g} \overrightarrow{g r a d} \hat{H}\right)}{k^{2} C C_{g} \hat{H}}
$$

Le paramètre de diffraction $\delta_{H}$ modifie les caractéristiques des vagues à savoir 
le nombre d'onde, la vitesse de phase et la vitesse de groupe puisqu'il vient :

$$
k^{\prime}=k \sqrt{1+\delta_{H}}, \quad C^{\prime}=C / \sqrt{1+\delta_{H}}, \quad C_{g}^{\prime}=C_{g} \sqrt{1+\delta_{H}}
$$

L'expression du paramètre de diffraction en coordonnées curvilignes s'écrit :

$$
\delta_{H}=\frac{\left\{\begin{array}{l}
\frac{C C g}{e_{1}^{2}} \frac{\partial^{2} \hat{H}}{\partial \xi^{2}}+\frac{C C g}{e_{2}^{2}} \frac{\partial^{2} \hat{H}}{\partial \eta^{2}} \\
+\left[\frac{C C_{g}}{e_{1} e_{2}} \frac{\partial}{\partial \xi}\left(\frac{e_{2}}{e_{1}}\right)+\frac{1}{e_{1}^{2}}\left(\frac{\partial C C_{g}}{\partial \xi}\right)\right] \frac{\partial \hat{H}}{\partial \xi}+\left[\frac{C C_{g}}{e_{1} e_{2}} \frac{\partial}{\partial \eta}\left(\frac{e_{1}}{e_{2}}\right)+\frac{1}{e_{2}^{2}}\right] \frac{\partial \hat{H}}{\partial \eta}
\end{array}\right\}}{k^{2} C C_{g} \hat{H}}
$$

L'équation de propagation de l'enveloppe de la houle en coordonnées curvilignes orthogonales en tenant compte des effets de diffraction devient :

$$
\begin{aligned}
& \frac{C C g}{e_{1}^{2}} \frac{\partial^{2} \hat{H}}{\partial \xi^{2}}+\frac{C C g}{e_{2}^{2}} \frac{\partial^{2} \hat{H}}{\partial \eta^{2}} \\
& +\left[2 i k^{\prime} e_{1} \frac{C C_{g}}{e_{1}^{2}}+\frac{C C_{g}}{e_{1} e_{2}} \frac{\partial}{\partial \xi}\left(\frac{e_{2}}{e_{1}}\right)+\frac{1}{e_{1}^{2}}\left(\frac{\partial C C_{g}}{\partial \xi}-g \frac{\partial \zeta}{\partial \xi}\right)\right] \frac{\partial \hat{H}}{\partial \xi} \\
& +\left[\frac{C C_{g}}{e_{1} e_{2}} \frac{\partial}{\partial \eta}\left(\frac{e_{1}}{e_{2}}\right)+\frac{1}{e_{2}^{2}}\left(\frac{\partial C C_{g}}{\partial \eta}-g \frac{\partial \zeta}{\partial \eta}\right)\right] \frac{\partial \hat{H}}{\partial \eta} \\
& +\left[\left[\left(1+i v_{t}\right) k^{2}-k^{\prime 2}\right] C C_{g}+\frac{i C C_{g}}{e_{1}^{2}}\left(\frac{\partial k^{\prime}}{\partial \xi} e_{1}+k^{\prime} \frac{\partial e_{1}}{\partial \xi}\right)+\right] \hat{H}=0 \\
& i k^{\prime} e_{1}\left(\frac{C C_{g}}{e_{1} e_{2}} \frac{\partial}{\partial \xi} \frac{e_{2}}{e_{1}}\right)+\frac{1}{e_{1}^{2}}\left(\frac{\partial C C_{g}}{\partial \xi}-g \frac{\partial \zeta}{\partial \xi}\right)
\end{aligned}
$$

\section{$4 \quad$ Résolution de l'équation elliptique}

La résolution de l'équation elliptique (15) est réalisée à l'avancement après discrétisation d'un système d'équations linéaires tridiagonales, obtenu en substituant l'expression des dérivées de $\hat{H}$ suivant $\eta$ à partir du développement en série de MacLaurin dans l'équation (15), et en adoptant une approximation de type Newmark pour la fonction $\hat{H}$ et sa dérivée suivant $\xi$. Le système, dont le second membre dépend de la solution et de ses dérivées des deux pas d'espace précédents, permet de déterminer la dérivée seconde de $\hat{H}$, la dérivée première, puis $\hat{H}$ sous forme complexe (BOUGIS, 2004). 


\section{$5 \quad$ Equation des raies}

La nouvelle équation eïkonale (9) permet d'établir le tracé des rayons de la houle lors de sa propagation du large vers la côte, en tenant compte de la modification du nombre d'onde dans les équations (13) lorsque la diffraction devient importante. En considérant $c=\omega / k^{\prime}$ avec $\omega$ constant, on admet que l'angle $\theta$ de courbure des rayons, suivant les abscisses et ordonnées curvilignes $n, s$ est défini par :

$$
\frac{\partial \theta}{\partial s}=-\frac{1}{c} \frac{\partial c}{\partial n}=\frac{1}{k^{\prime}} \frac{\partial k^{\prime}}{\partial n}
$$

Après développement et en absence de courant, l'équation des raies en tenant compte du paramètre de diffraction $\delta_{H}$ devient (HOLTUIJSEN et al., 2003):

$$
\frac{\partial \theta}{\partial s}=\underbrace{\frac{1}{k} \frac{\partial k}{\partial n}}_{\text {réfraction }}+\underbrace{\frac{1}{2\left(1+\delta_{H}\right)} \frac{\partial \delta_{H}}{\partial n}}_{\text {diffraction }}
$$

Dans le cas où la diffraction est nulle, on retrouve bien l'équation appliquée classiquement en réfraction pure.

\section{$6 \quad$ Construction de la métrique}

La longueur de la crête de départ ainsi que sa direction sont choisies libre sur la grille bathymétrique. La position de chaque crête dans la propagation de la houle est calculée à l'avancement en réfraction pure dans un premier temps, suivant l'équation eïkonale en admettant $k^{\prime}=k$. Le calcul du paramètre de diffraction $\delta_{H}$, qui dépend des variations de $\hat{H}$ et de ses dérivées, intervient ensuite pour modifier les caractéristiques des vagues. L'équation complexe (15) admettant une solution réelle que si le sinus de la phase est nul, c'est-à-dire sur les crêtes, l'opération est réitérée et le maillage modifié en tenant compte du paramètre de diffraction. Le modèle converge lorsque la valeur du paramètre de diffraction se stabilise et que la partie imaginaire de l'expression complexe de $\hat{H}$ s'annule.

\section{$7 \quad$ Condition aux limites}

Les dérivées premières et secondes de la hauteur sont conservées le long des rayons latéraux. Lorsque les rayons sont arrêtés par une île, deux nappes prennent naissances de part et d'autre et sont traitées indépendamment. Au contraire lorsque la diffraction empêche le croisement, les crêtes tendent suivant les cas, à se déphaser voir à se détacher, puis à se raccrocher une fois le haut fond passé. 


\section{$8 \quad$ Exemples d'applications}

\subsection{L'expérience de Vincent et Briggs}

Le modèle a été testé sur le haut fond elliptique de l'expérience de VINCENT \& BRIGGS, 1989. Il s'agit d'une bosse d'une longueur de $8 \mathrm{~m}$ et d'une largeur de $6 \mathrm{~m}$ pour une hauteur d'environ $30 \mathrm{~cm}$, posée sur un fond plat (figure 1), pour une houle monochromatique incidente de hauteur $\mathrm{H}=2.5 \mathrm{~cm}$ et de période $\mathrm{T}=1.3 \mathrm{~s}$ :

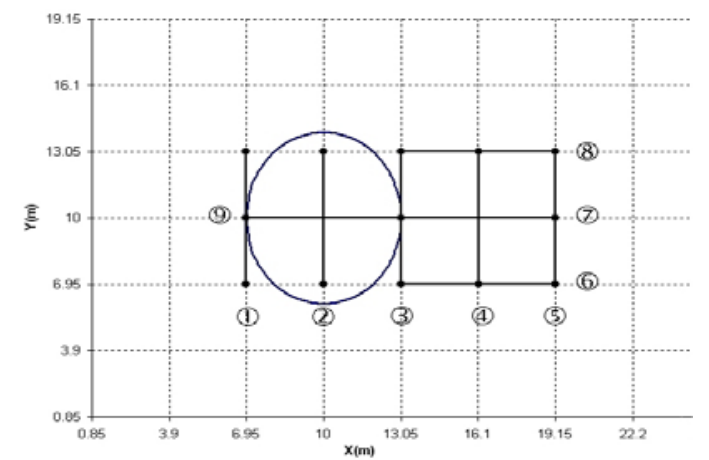

Figure 1 : Dispositif expérimental de VINCENT \& BRIGGS, 1989. La houle arrive par la gauche et les sections numérotées représentent les points de mesure

Si aucune itération n'est opérée dans le modèle, les résultats sont obtenus en réfraction pure, ce qui permet une comparaison avec la réfraction-diffraction où plusieurs itérations sont nécessaires pour atteindre la convergence du modèle.
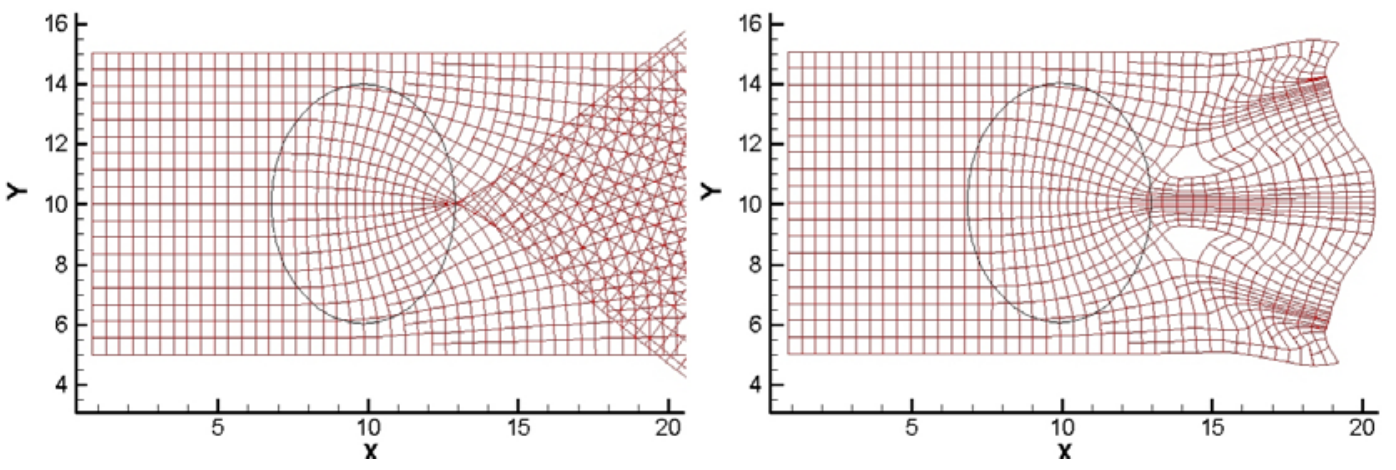

Figure 2 : Comparaison du plan de vagues en réfraction pure à gauche, et en réfraction-diffraction à droite (pas d'espace $=0.2$ longueur d'ondes)

On remarque sur la figure 2 l'obtention d'une caustique en réfraction pure et le croisement de 2 nappes de part et d'autre. En revanche en réfraction-diffraction, le paramètre de diffraction modifie les caractéristiques des vagues et notamment la longueur d'onde qui tend à s'allonger là ou la hauteur est importante et à diminuer là où les hauteurs sont faibles. Le paramètre de diffraction influe également sur la 
direction de propagation de la houle à travers l'équation (17). La diffraction opère ainsi afin d'éviter aux rayons de se croiser. Néanmoins la houle ayant extrêmement ralenti sur le sommet de la bosse, il semble que les crêtes subissent un "décrochage" et un déphasage. Ce problème sera sujet à de futures investigations en bassin à houle sur le même type de bosse.

SECTION 3

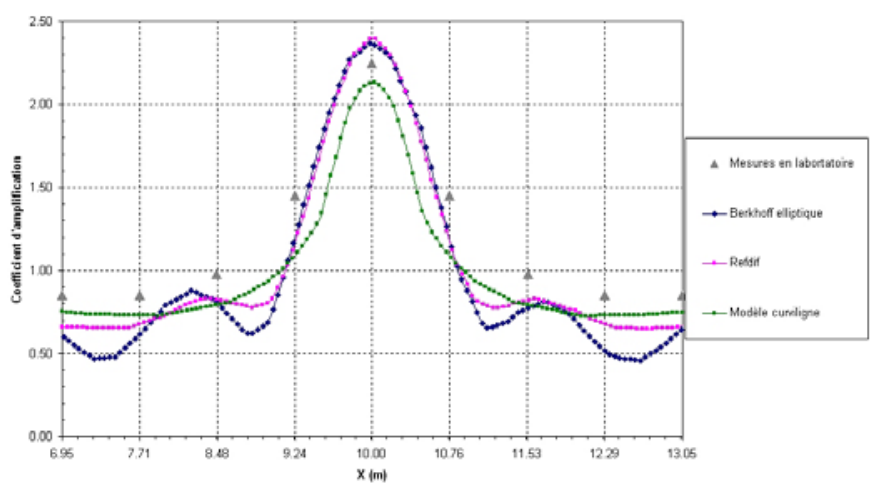

Figure 3 : Comparaison des hauteurs de houle sur la section 3 entre mesures en laboratoire, modèle elliptique (bleu), RefDif (rose) et modèle curviligne(vert)

Les hauteurs de houle en réfraction-diffraction ont ensuite été comparées au modèle parabolique RefDif (KIRBY \& DALRYMPLE, 1983), ainsi qu'à un modèle elliptique (Berkhoff complet), sur la section 3 de l'expérience c'est-à-dire au niveau de la caustique obtenue en réfraction pure. Les résultats (figure 3) montrent une légère sous-estimation de la hauteur maximale de la houle au centre, mais de meilleurs résultats que Refdif et que le modèle elliptique sur les côtés, même si ceux-ci restent légèrement inférieurs aux valeurs expérimentales.

\subsection{Rade de Marseille}

Le modèle en coordonnées curviligne a été confronté aux résultats de SWAN sur la rade de Marseille. Pour pouvoir comparer le modèle à résolution de phase au modèle spectral, le calcul est d'abord effectué en admettant la houle monochromatique et monodirectionnelle. Les simulations sont reproduites sur une large plage de fréquences et de directions puis recombinées afin d'extraire les valeurs statistiques de houle. Le spectre fréquentiel est un spectre classique de JONSWAP avec un coefficient d'élancement $\gamma$ de 3,3. La densité d'énergie $S_{0}$ de la houle incidente est alors répartie comme suit sur la bande de fréquences:

$S_{0}(\omega)=a H_{S 0}^{2} \omega_{P}^{4} \omega^{-5} e^{-1,25\left(\frac{\omega}{\omega_{P}}\right)^{-4}} \gamma^{e^{-\frac{\left(\omega-\omega_{P}\right)^{2}}{2 \sigma^{2} \omega_{P}^{2}}}}$ 
Où a est une constante telle que la hauteur significative $H_{S}$ puisse vérifier la loi classique inhérente aux processus supposés gaussiens (et dont les maximaux suivent une loi de Rayleigh) :

$$
H_{S_{0}}=4 \sqrt{\int_{0}^{\infty} S_{0}(\omega) d \omega}
$$

La distribution directionnelle de l'énergie de la houle incidente est issue de la théorie simple : spectre directionnel tel que $D_{\theta}=b \cos ^{4}\left(\theta-\theta_{\text {moyen }}\right)$ où $\mathrm{b}$ est une constante telle que $\int_{-\pi}^{\pi} D_{\theta} \mathrm{d} \theta=1$, et $\theta$ la direction.

Les valeurs de hauteurs significatives de SWAN et du modèle curviligne pour la direction $\mathrm{N} 240^{\circ}$ ont ensuite été interpolées au devant de la digue du large (figure 4) pour une conditions de houle centennale. Les résultats montrent une très bonne corrélation entre les deux modèles.

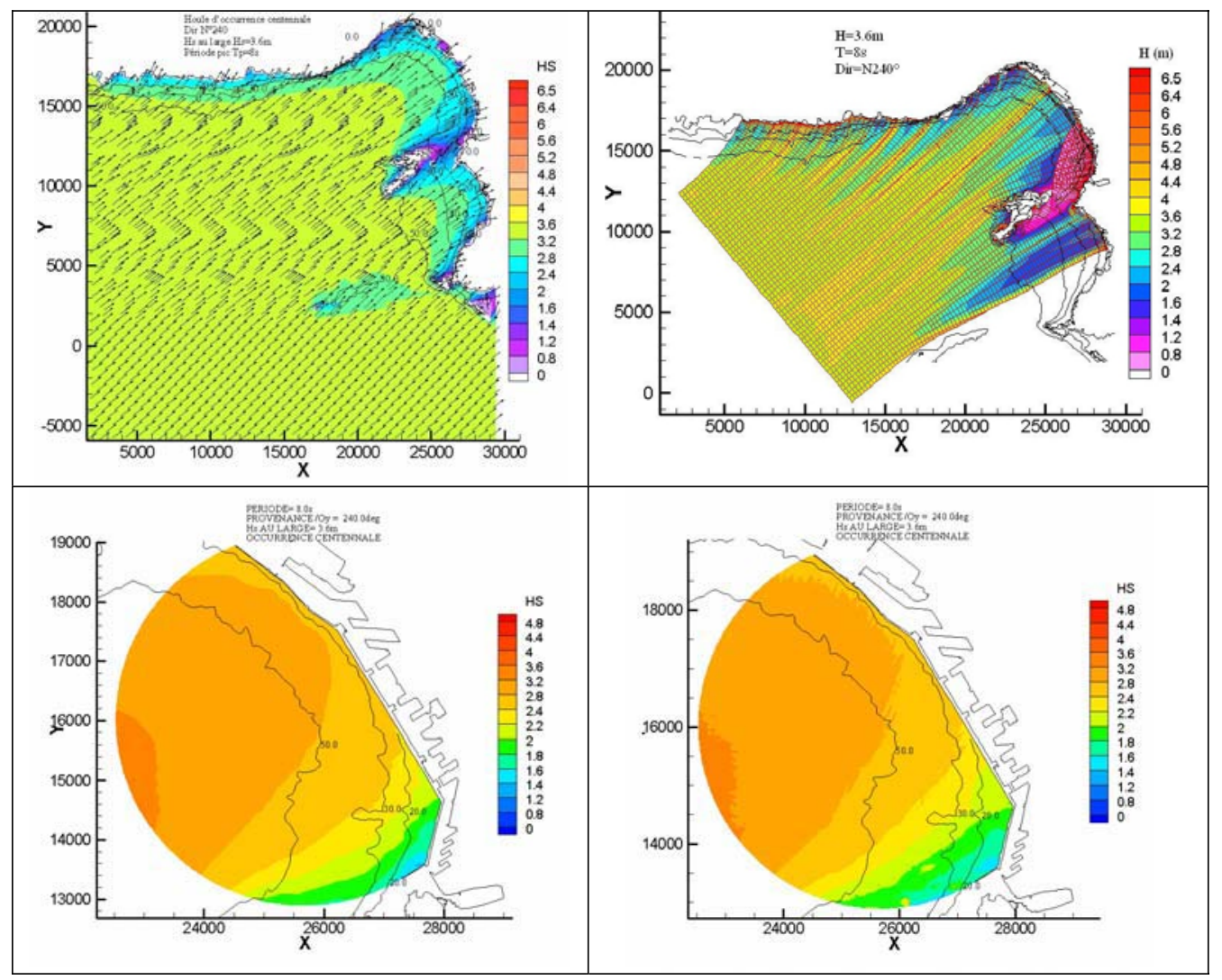

Figure 4 : Comparaison des plans de vagues et des hauteurs significatives interpolées entre SWAN à gauche, et le modèle curviligne à droite, pour une condition de houle centennale de $N 240^{\circ}(\mathrm{Hs}=3.6 \mathrm{~m}$ et $\mathrm{Tp}=8 \mathrm{~s})$ 


\section{Conclusion}

L'insertion d'un paramètre de diffraction dans un modèle d'approche de la houle en coordonnées curvilignes permet de mieux prendre en considération cet effet, et d'étendre le champ de validité du modèle sur des bathymétries complexes. Il met également en avant l'influence de la diffraction lorsque la houle passe au dessus d'un haut fond, qui modifie les caractéristiques des vagues là où la convergence est trop forte et évite ainsi caustiques et croisements de nappes.

Cette constatation révèle que la connaissance du comportement de la houle derrière un haut-fond n'est pas tout à fait maîtrisée. C'est pourquoi des recherches plus approfondies sur cet aspect devraient avoir lieu au travers d'essais en bassin à houle, pour permettre une meilleure gestion des conditions aux limites et favoriser la robustesse du modèle.

Néanmoins le modèle s'applique déjà de manière satisfaisante aussi bien dans des baies, qu'au dessus de certains hauts fonds ou derrière des îles, sans limitations angulaires et avec une grande rapidité de calcul.

Un tel modèle permet ainsi de suivre la propagation de la houle du large vers la côte en prenant en compte les effets de réfraction diffraction, la présence de courant et les effets de dissipation, pour des houles régulières et irrégulières, tout en ayant l'avantage de conserver la phase dans le calcul. Il peut donc être couplé à un modèle d'agitation portuaire ou à un modèle hydro sédimentaire.

\section{$10 \quad$ Références}

1 BERKHOFF J.C.W. (1972). Computation of combined refractiondiffraction. Proc. $13^{\text {th }}$ Coastal Eng. Conf., ASCE, pp 471-490.

${ }^{2}$ BOUGIS J. (2004). Modèle d'approche de la houle par une méthode de refraction-diffraction en coordonnées curvilignes. VIII ${ }^{\text {èmes }}$ Journées Nationales Génie Côtier - Génie Civil, Compiègne, pp 53 -62.

3 HOLTHUIJSEN L.H., HERMAN A., BOOIJ N., (2003). Phasedecoupled refraction-diffraction for spectral wave models. Coastal Engineering 49, pp 291-305

4 KIRBY J.T., DALRYMPLE R.A. (1983). A parabolic equation for combined refraction and diffraction of stokes waves by mildly varying topography. Journal of Fluid Mechanics 136, pp 453-466

5 RADDER A.C. (1979). On the parabolic equation method for waterwave propagation. Journal of Fluid Mechanics 95, pp 159-176.

6 VINCENT C.L., BRIGGS M.J. (1989). Refraction-diffraction of irregular waves over a mound. J. Waterw. Port Coast. Ocean Eng. 115, pp 269-284 